\title{
A Quantitative Model of Human Jejunal Smooth Muscle Cell Electrophysiology
}

\author{
Weiwei $\mathrm{Ai}^{{ }^{*}}$ and David P Nickerson ${ }^{1}$ \\ ${ }^{1}$ Auckland Bioengineering Institute, University of Auckland, New Zealand
}

\section{ORIGINAL}

\begin{abstract}
The Poh et al. (2012) paper describes the first biophysically based computational model of human jejunal smooth muscle cell (hJSMC) electrophysiology. The ionic currents are described by either a traditional Hodgkin-Huxley $(\mathrm{HH})$ formalism or a deterministic multi-state Markov (MM) formalism. We create a modularized CellML implementation of the model, which is able to reproduce clamping behaviours of individual currents and whole cell action potential traces. In addition, some inconsistencies have been uncovered and discussed in this paper.
\end{abstract}

Keywords: Human jejunal smooth muscle cell, computational model, CellML

\section{Curated Model Implementation}

http://doi.org/10.36903/physiome.16590317

Primary Publications

Y. C. Poh, A. Corrias, N. Cheng, and M. L. Buist. A quantitative model of human jejunal smooth muscle cell electrophysiology. 2012.

OPEN ACCESS

Reproducible Model

Edited by

Shelley Fong

Curated by

Anand Rampadarath

${ }^{*}$ Corresponding author weiwei.ai@auckland.ac.nz

Submitted 09 Aug 2021

Accepted 09 Sept 2021

Citation

Ai and Nickerson

A Quantitative Model of

Human Jejunal Smooth

Muscle Cell

Electrophysiology. Physiome.

doi: $10.36903 /$ phys-

iome.16590317

\section{Introduction}

In the primary paper (Poh et al., 2012), the first biophysically based model of human jejunal smooth muscle cell (hJSMC) electrophysiology was presented. Here, we divide the mathematical model into distinct sub-modules encoded in CellML enabling reuse of the various sub-modules in future studies and models. We attempt to reproduce individual ionic currents, cellular voltage behaviour and sensitive analysis corresponding to changes of channel conductance. From the primary paper we extracted data using the Engauge digitizer software (Mitchell et al., 2020) to compare the current simulation results against those in the primary publication. In doing so, we found inconsistencies in parameter values and mathematical equations presented in the primary paper, as well as the simulation experiment settings. These discrepancies are discussed in section 4.

\section{Model description}

\subsection{Primary Publication}

In the hJSMC model (Poh et al., 2012), L-type calcium channels, the large conductance calcium and voltage activated potassium channels (BK) channels, and sodium channels are formulated using a deterministic multi-state Markov (MM) model (Sakmann and Neher, 1996), while the T-type calcium channels and voltage dependent potassium current employ a traditional Hodgkin-Huxley $(\mathrm{HH})$ formalism (Hodgkin and Huxley, 1952). The sodium calcium exchanger and sodium potassium pump formulation are based on the work proposed by ten Tusscher et al. (2004). The primary publication shows that the model has been validated against a wide range of experiments and data. The authors also provide CellML code https://models.physiomeproject.org/w/yc_poh/poh_ 
2012 and C code https://computationalbiolab.github.io/jejunal_smc_model/. The mathematical descriptions in the publication (Poh et al., 2012) along with these codes are the basis to build the curated CellML implementation presented here.

\subsection{Modularized CellML model}

The modularized version of the CellML implementation is available on Physiome Model Repository (PMR) at https://models.physiomeproject.org/workspace/692 and the documentation can be found in the corresponding exposure at https://models.physiomeproject.org/e/6c7. In this manuscript we focus on reproducibility and reusability. The main components of this model and the performed simulation experiments are summarized as follows.

The membrane potential component defines the complete equations of the membrane potential and total ionic current. It combines the imported ionic current components and ionic concentrations components. The component can be stimulated by various periodic stimuli and generates a sequence of action potentials. The clamped current component defines the complete equations of the total and individual ionic currents, when performing voltage or concentration clamp simulation experiments.

The individual ionic current components share the same formulation $I=g_{\max } P O\left(V_{m}-E\right)$, where $V_{m}$ is the membrane potential and $E=\frac{R T}{z F} \ln \left(\frac{X_{0}}{X_{i}}\right)$ is the reversal potential defined in the Nernst potential component. The open probability $P O$ computation depends on the formalism. For L-type calcium channels, the large conductance calcium and voltage activated potassium channels (BK) channels, sodium channels, a deterministic multi-state Markov (MM) model (Sakmann and Neher, 1996) is used to describe various channel states. Such currents incorporate the channel states components. The Hodgkin-Huxley $(\mathrm{HH})$ formalism of the T-type calcium channels and voltage dependent potassium current includes the gating kinetics components along with steady state of gates and time constants components. The sodium calcium exchanger and sodium potassium pump formulation is different from the aforementioned currents, which is defined in its own component.

The ionic concentrations component defines the dependence of the intracellular concentrations on the membrane currents. The temperature factor component encodes the temperature coefficient $\phi=Q_{10}^{\frac{T-T_{0}}{10}}$, where $Q_{10}$ is species specific parameter, $T_{0}$ is the reference temperature (i.e., the model construction temperature), and $T$ is the desired temperature for a given simulation experiment. This coefficient is included in the equations listed in Table 3.

Following best practices, our CellML implementation separates the mathematics from the parameterisation of the model. The mathematical model is imported into a specific parameterised instance in order to perform numerical simulations. The parameterisation would include defining the stimulus protocol to be applied. We have three sets of simulation experiments and corresponding simulation results to reproduce the corresponding figures in the primary publication:

1. the patch clamp experiment is used to validate the individual ionic currents;

2. the periodic stimulation experiment is used to generate a sequence of action potentials; and

3. the sensitivity analysis experiment is used to evaluate the contributions of currents to the membrane voltage.

Simulation settings and detailed solver information are encoded in SED-ML documents for execution of the simulation experiments (Waltemath et al., 2011). The Python scripts used with OpenCOR (Garny and Hunter, 2015) to perform simulations and produce the figures in the primary paper are also included in the folder <Simulation>. The name of the simulation and plot scripts indicate the figure number in the primary paper. For example, Fig2_sim.py is used to generate the simulation data and Fig2_plot.py reproduces the graph shown in Figure 2 from Poh et al. (2012). 


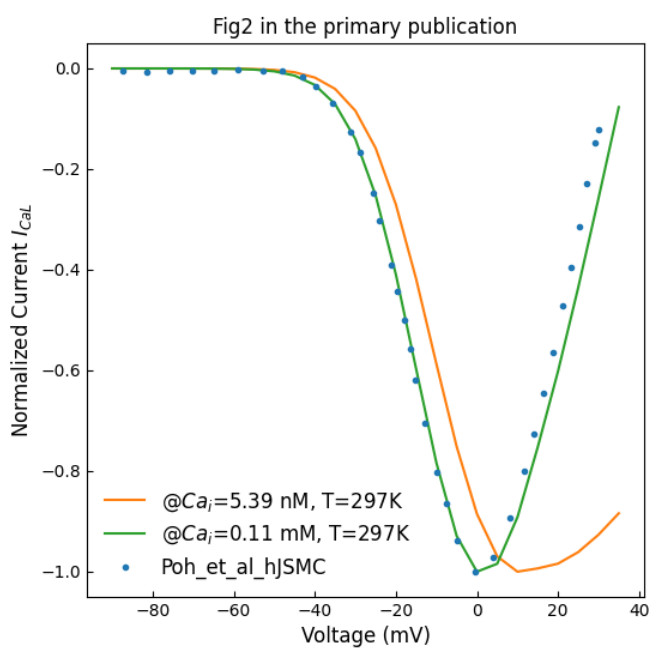

Figure 1. Normalized L-type $C a^{2+}$ channels peak I-V plot (c.f., Fig. 2 in Poh et al. (2012)).

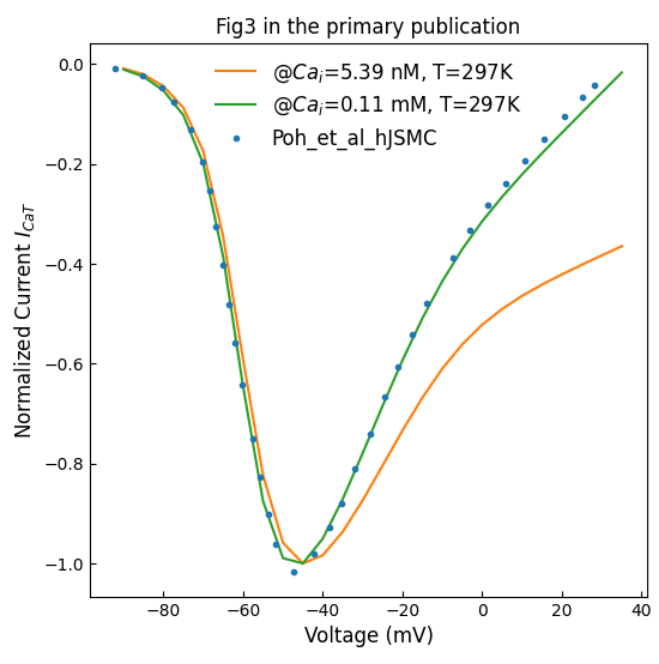

Figure 2. Normalized T-type $\mathrm{Ca}^{2+}$ channels peak I-V plot (c.f., Fig. 3 in Poh et al. (2012)).

\section{Model results}

\subsection{Response of individual ionic currents to clamped voltage}

In the patch clamp experiment, the clamped current component is configured and parameterised with an applied patch clamp protocol. The clamping parameters can be changed by the user, while the values used in this article are listed in Table 1. The holding voltage duration is 1 second and the temperature is 297 Kelvin. The simulation experiment can be performed by loading the corresponding SED-ML document into OpenCOR and executing the simulation. By increasing the intracellular concentrations, we have reproduced the IV curves in Figures 1, 2 and 5, however, the specific experiment settings cannot be confirmed by the authors.

Table 1. Clamping parameters

\begin{tabular}{ccccc}
\hline Fig & Holding voltage $(\mathrm{mV})$ & Test voltages $(\mathrm{mV})$ & Current & $X_{i}(\mathrm{mM})$ \\
\hline 2 & -100 & $-90: 5: 40$ & $I_{C a L}$ & $C a_{i}^{2+}=5.388 e-5, C a_{i}^{2+}=0.11$ \\
3 & -100 & $-90: 5: 40$ & $I_{C a T}$ & $C a_{i}^{2+}=5.388 e-5, C a_{i}^{2+}=0.11$ \\
4 & -70 & $-70: 5:-15$ & $I_{K v}$ & $K_{i}^{+}=153.6$ \\
5 & -70 & $-70: 10: 70$ & $I_{B K}$ & $K_{i}^{+}=153.6, C a_{i}^{2+}=0.001, C a_{i}^{2+}=0.0003$ \\
6 & -100 & $-90: 5: 30$ & $I_{N a}$ & $N a_{i}^{+}=10.57, N a_{i}^{+}=50$ \\
\hline
\end{tabular}

In the presented figures, the dots denote the simulated data extracted from the primary publication, while the solid lines are the simulation results produced by the current CellML implementation.

Figure 1 shows the normalized L-type $C a^{2+}$ channels peak I-V plot with different intracelluar concentrations. During simulation, the $\theta$ and $\delta$ in Equations S-33 and S-34 are set to 0 to switch off the $\mathrm{Ca}_{i}^{2+}$ dependency. Figure 2 shows normalized T-type $C a^{2+}$ channels peak I-V plot.

Figure 3 shows normalized I-V plot of whole cell voltage-activated potassium currents. The holding voltage was not specified in the primary paper, and we assume here a value of $-70 \mathrm{mV}$.

Figure 4 shows open probability of BK channel versus clamping voltage at various intracellular calcium concentrations.

Figure 5 shows normalized sodium channel peak I-V plot various intracellular $\mathrm{Na}^{+}$concentrations.

Figure 6 shows whole cell normalized I-V data from hJSMC model under near calcium-free conditions. The intracellular $\mathrm{Na}^{+}$concentration is set to $10.57 \mathrm{mM}$, while the value is unknown 


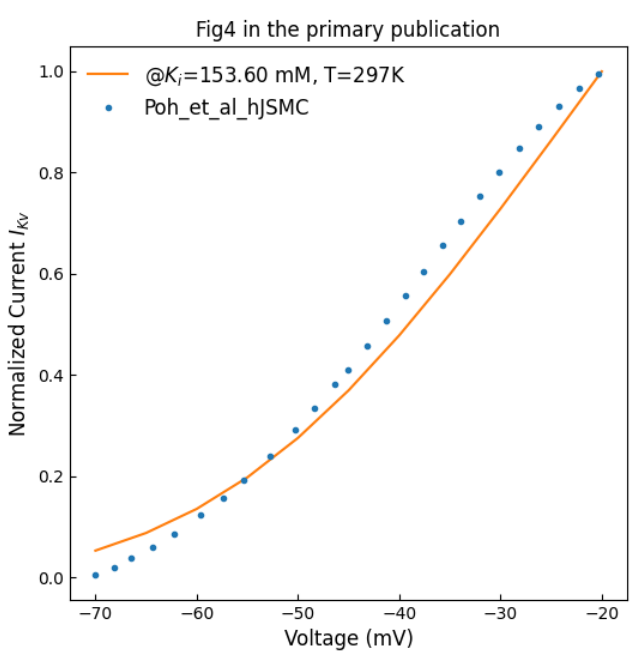

Figure 3. Normalized I-V plot of whole cell voltage-activated potassium currents (c.f., Fig. 4 in Poh et al. (2012)).

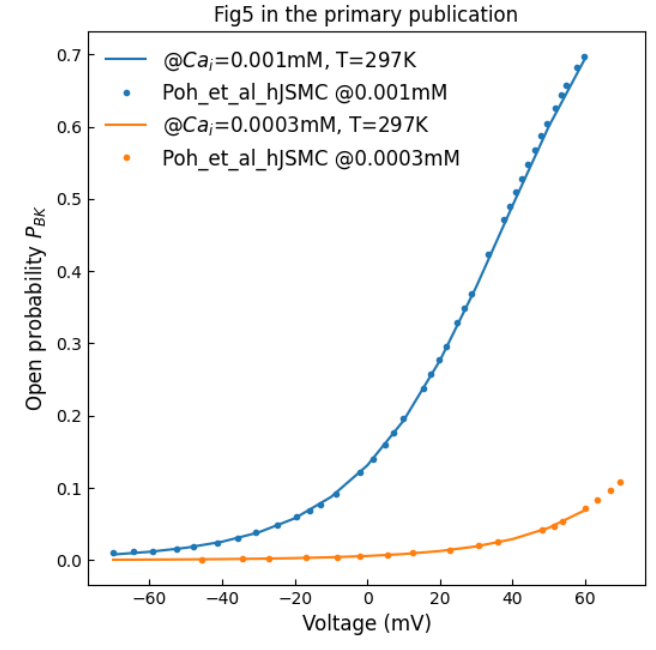

Figure 4. Open probability versus clamping voltage plots, across various free intracellular $\mathrm{Ca}^{2+}$ concentrations (c.f., Fig. 5 in Poh et al. (2012)).

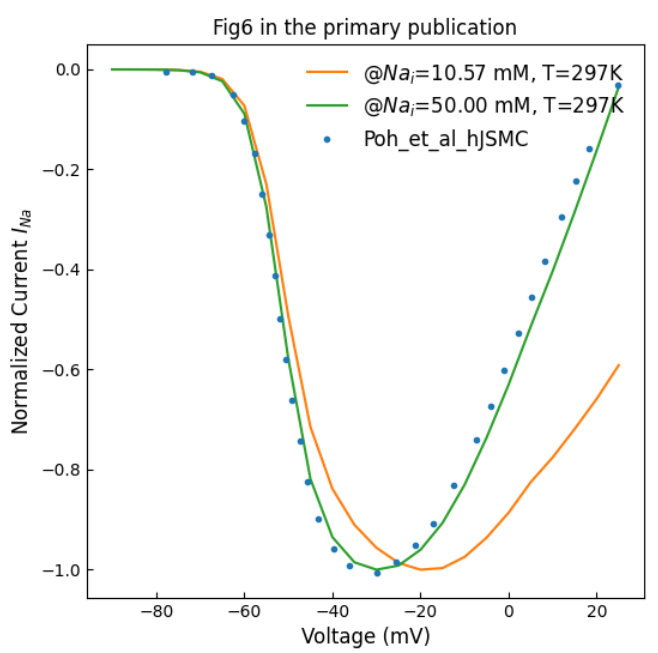

Figure 5. Normalized $\mathrm{Na}^{+}$channel peak I-V plot (c.f., Fig. 6 in Poh et al. (2012)).

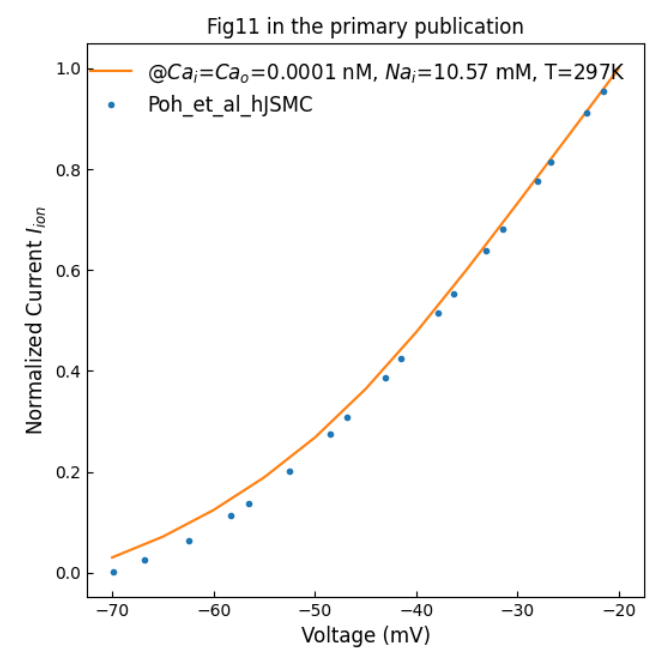

Figure 6. Simulated whole cell normalized I-V data under near calcium-free conditions (c.f., Fig. 11 in Poh et al. (2012)). 
in the primary publication.

\subsection{Simulated action potentials}

In the periodic stimulation experiment, the membrane potential component is configured and parameterised with a periodic stimulus current. The parameters of stimulation can be changed and the following simulation uses 310 Kelvin for the temperature setting.

Figure 7 shows the simulated hJSMC action potential trace and free intracellular calcium concentration after a simulation of 30 minutes of electrical activity.
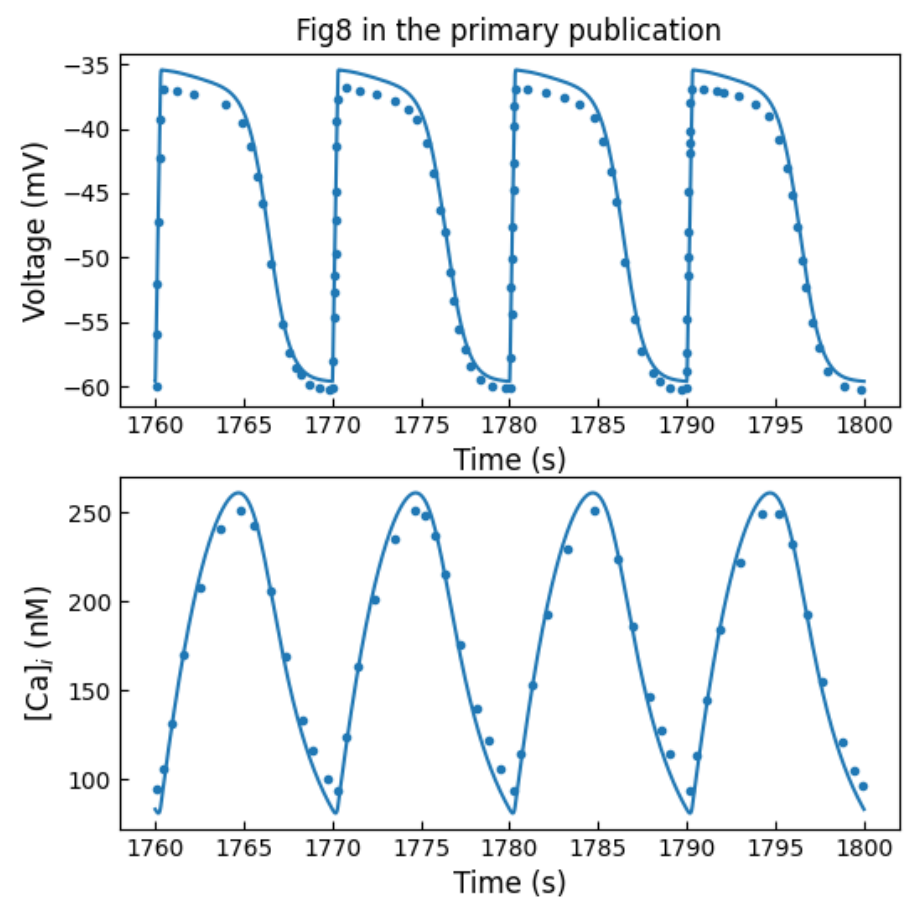

Figure 7. simulated hJSMC action potential trace and free intracellular calcium concentration after a simulation of 30 minutes of electrical activity (c.f., Fig. 8 in Poh et al. (2012)).

\subsection{Sensitivity Analysis}

In the sensitivity analysis experiment, the membrane potential component is configured to accept the changes of maximum conductance of ionic channels in the component g_parameters.

Figure 8 shows sensitivity analysis by $50 \%$ increase or decrease in maximum channel conductance. This evaluates the contributions of key ionic currents towards hJSMC membrane voltage. The last plot shows the free intracellular $\mathrm{Ca}^{2+}$ concentrations corresponding to changes in $I_{C a L}$.

\section{Discussion}

Based on the author provided model implementation (Poh et al., 2012), we modularized the CellML implementation for reusability. Additionally, we added clamped current component, patch clamp protocol and voltage clamp experiment to simulate ionic currents during a voltage clamp. We also modified the periodic current protocol and periodic stimulation experiment to enable the periodic stimulation for Figure 8.

During the curating process, we found some parameters (see Table 2) and equations (see Table 3 ) which are inconsistent from the ones presented in the primary publication. 

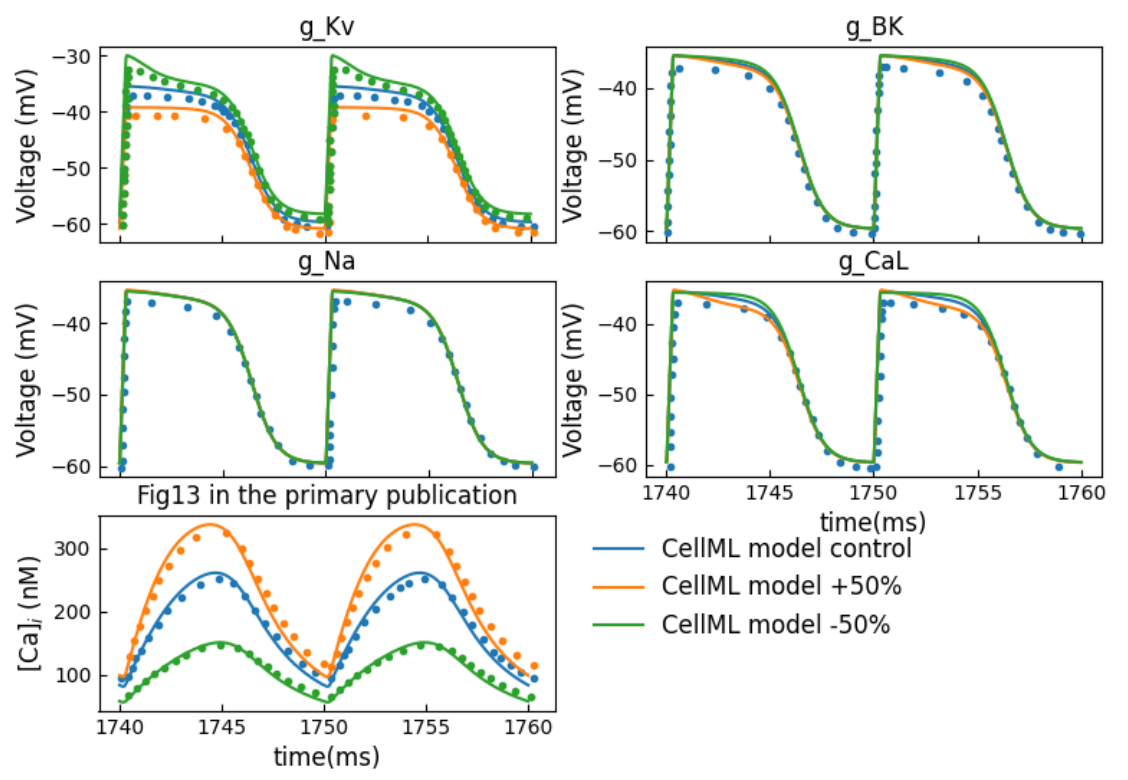

- CellML model control

- CellML model $+50 \%$

- CellML model $-50 \%$

Figure 8 . Sensitivity analysis by $50 \%$ increase or decrease in maximum channel conductance (c.f., Fig. 13 in Poh et al. (2012)).

Since the clamping values for intracellular concentrations of $\mathrm{Ca}_{i}^{2+}, \mathrm{Na}_{i}^{+}$and $\mathrm{K}_{i}^{+}$were not specified in the paper, we used the initial values presented in the original CellML model in the first attempt. However, the difference becomes significant at less negative clamping voltages when reproducing the original Figures 2, 3, and 6. We then increased the intracellular concentrations for a better fit. However, the values of intracellular concentrations in particular $\mathrm{Ca}_{i}^{2+}$ are quite larger than physiological value, which should be aware of in future usage of this model.

Table 2. Inconsistency of parameters

\begin{tabular}{lccrr}
\hline Parameters & primary paper & Original CellML & C code & current CellML \\
\hline$P_{N C X}$ & 39.8437 & 1992.335 & 1992.1865 & 1992.1865 \\
$P_{N a K}$ & 0.1852 & 9.26 & 16.197 & 16.197 \\
$\tau_{d C a t}$ & 1.9058 & 1.9058 & 1.9508 & 1.9508 \\
1st factor in Eq(S-24) & 0.05956 & 0.05956 & 0.005956 & 0.005956 \\
\hline
\end{tabular}


Table 3. Inconsistency of equations

\begin{tabular}{|c|c|c|c|c|}
\hline Equations & $\begin{array}{l}\text { primary } \\
\text { paper }\end{array}$ & $\begin{array}{l}\text { Original } \\
\text { CellML }\end{array}$ & C code & current CellML \\
\hline$S-5, S-6, S-7$ & $\begin{array}{l}\text { without unit } \\
\text { conversion }\end{array}$ & $\times 1 e-15$ & $\begin{array}{l}V_{\text {cell }} \text { in } \mathrm{mm}^{3} \\
\text { and } \times 1 e-9\end{array}$ & $\times 1 e-15$ \\
\hline S-13, S-14 & No $T^{1}$ & with $T^{2}$ & with $\mathrm{T}^{2}$ & with $\mathrm{T}^{2}$ and $T_{0}=310 \mathrm{~K}$ \\
\hline$S-23, \ldots, S-28$ & No $T^{1}$ & with $\mathrm{T}^{2}$ & with $\mathrm{T}^{2}$ & with $\mathrm{T}^{2}$ and $T_{0}=310 \mathrm{~K}$ \\
\hline S-33, S-34 & No $T^{1}$ & with $\mathrm{T}^{2}$ & with $\mathrm{T}^{2}$ & with $\mathrm{T}^{2}$ and $T_{0}=310 \mathrm{~K}$ \\
\hline S-36, S-37, & No $T^{1}$ & with $\mathrm{T}^{2}$ & with $\mathrm{T}^{2}$ & with $\mathrm{T}^{2}$ and $T_{0}=297 \mathrm{~K}$ \\
\hline$S-43, S-44$ & No $T^{1}$ & with $\mathrm{T}^{2}$ & with $\mathrm{T}^{2}$ & with $\mathrm{T}^{2}$ and $T_{0}=297 \mathrm{~K}$ \\
\hline$S-80, \ldots, S-91$ & No $T^{1}$ & with $\mathrm{T}^{2}$ & with $\mathrm{T}^{2}$ & with $\mathrm{T}^{2}$ and $T_{0}=297 \mathrm{~K}$ \\
\hline$S-67, \ldots, S-70$ & with $C a_{j}^{2+}$ & without $C a_{j}^{2+}$ & without $C a_{i}^{2+}$ & without $\mathrm{Ca}_{j}^{2+}$ \\
\hline$S-75, \ldots, S-78$ & with $C a_{i}^{2+}$ & without $C a_{i}^{2+}$ & without $C a_{i}^{2+}$ & without $C a_{i}^{2+}$ \\
\hline
\end{tabular}

${ }^{1}$ No temperature correction.

2 Multiplied by corresponding temperature factor $\phi=Q_{10}^{\frac{T-T_{0}}{10}}$.

\section{Acknowledgements}

The authors gratefully acknowledge the assistance of Alberto Corrias and Martin Buist in the validation of the model implementation presented here.

\section{References}

A. Garny and P. J. Hunter. OpenCOR: a modular and interoperable approach to computational biology. Front Physiol, 6, 2015. doi: 10.3389/fphys.2015.00026. URL http://journal.frontiersin. org/article/10.3389/fphys.2015.00026/abstract.

A. L. Hodgkin and A. F. Huxley. A quantitative description of membrane current and its application to conduction and excitation in nerve. The Journal of physiology, 117(4):500-544, 1952.

M. Mitchell, B. Muftakhidinov, T. Winchen, A. Wilms, B. v. Schaik, badshah400, Mo-Gul, T. G. Badger, Z. Jędrzejewski-Szmek, kensington, and kylesower. markummitchell/engauge-digitizer: Nonrelease, July 2020. URL https://doi.org/10.5281/zenodo.3941227.

Y. C. Poh, A. Corrias, N. Cheng, and M. L. Buist. A quantitative model of human jejunal smooth muscle cell electrophysiology. 2012.

B. Sakmann and E. Neher. Single-channel recording. General Pharmacology: The Vascular System, 27(6):1078, 1996. ISSN 0306-3623. doi: https://doi.org/10.1016/0306-3623(96)90073-7. URL https://www.sciencedirect.com/science/article/pii/0306362396900737.

K. H. ten Tusscher, D. Noble, P.-J. Noble, and A. V. Panfilov. A model for human ventricular tissue. American Journal of Physiology-Heart and Circulatory Physiology, 286(4):H1573-H1589, 2004.

D. Waltemath, R. Adams, F. T. Bergmann, M. Hucka, F. Kolpakov, A. K. Miller, I. I. Moraru, D. Nickerson, S. Sahle, J. L. Snoep, and N. L. Novère. Reproducible computational biology experiments with SED-ML - The Simulation Experiment Description Markup Language. BMC Systems Biology, 5(1):198, Dec. 2011. ISSN 1752-0509. doi: 10.1186/1752-0509-5-198. URL http://www.biomedcentral.com/1752-0509/5/198/abstract. 
Reproducibility report for: A Quantitative Model of Human Jejunal Smooth Muscle Cell Electrophysiology.

Submitted to: Physiome

Manuscript number/identifier: S000009

Curation outcome summary: Successfully reproduced all the figures presented in this manuscript.

Box 1: Criteria for repeatability and reproducibility

\section{Model source code provided:}

$\square$ Source code: a standard procedural language is used (e.g. MATLAB, Python, C)

$\square$ There are details/documentation on how the source code was compiled

$\square$ There are details on how to run the code in the provided documentation

$\square$ The initial conditions are provided for each of the simulations

$\square$ Details for creating reported graphical results from the simulation results

Source code: a declarative language is used (e.g. SBML, CelIML, NeuroML)

$\square$ The algorithms used are defined or cited in previous articles

$\square$ The algorithm parameters are defined

$\square$ Post-processing of the results are described in sufficient detail

\section{Executable model provided:}

$\square$ The model is executable without source (e.g. desktop application, compiled code, online service)

$\square$ There are sufficient details to repeat the required simulation experiments

\section{The model is described mathematically in the article(s):}

Equations representing the biological system

There are tables or lists of parameter values

There are tables or lists of initial conditions

Machine-readable tables of parameter values

Machine-readable tables of initial conditions

The simulation experiments using the model are described mathematically in the article:

$\square$ Integration algorithms used are defined

$\square$ Stochastic algorithms used are defined

$\square$ Random number generator algorithms used are defined

$\square$ Parameter fitting algorithms are defined

The paper indicates how the algorithms yield the desired output 
Box 2: Criteria for accessibility

Model/source code is available at a public repository or researcher's web site

$\square$ Prohibitive license provided

$\square$ Permissive license provided

Open-source license provided

All initial conditions and parameters are provided

All simulation experiments are fully defined (events listed, collection times and measurements specified, algorithms provided, simulator specified, etc.)

Box 3: Rules for Credible practice of Modeling and Simulation ${ }^{2}$

aModel credibility is assessed using the Interagency Modeling and Ananlysis Group conformance rubric: https://www.imagwiki.nibib.nih.gov/content/10-simple-rules-conformance-rubric

Define context clearly: Extensive

Use appropriate data: Extensive

Evaluate within context: Extensive

List limitations explicitly: Insufficient

Use version control: Extensive

Document adequately: Extensive

Conform to standards: Extensive

Box 4: Evaluation

Model and its simulations could be repeated using provided declarative or procedural code

Model and its simulations could be reproduced 
Summary comments: Model and source code are available in the associated OMEX archive. This was used in our attempt to reproduce the results presented in the paper. We successfully ran the python scripts provided to reproduce Figure 1 - Figure 8 as presented in this manuscript.

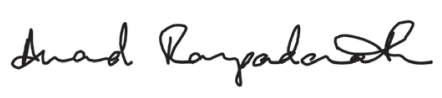

Anand K. Rampadarath ${ }^{1}$, PhD Curator

Center for Reproducible Biomedical Modeling Auckland Bioengineering Institute, University of Auckland

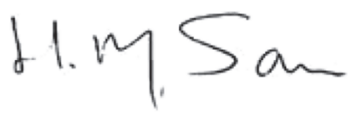

Herbert M. Sauro, Professor Director

Center for Reproducible Biomedical Modeling University of Washington, Seattle, WA

\footnotetext{
${ }^{1}$ Email: a.rampadarath@auckland.ac.nz
} 